\title{
Time-Resolved Small-Angle X-Ray Scattering Studies on the Melting Behavior of Poly(e-caprolactone)-block-Polybutadiene Copolymers
}

\author{
Shuichi NoJIMA, ${ }^{\dagger}$ Yukiyo Kanda, and Shintaro SaSaKI \\ School of Materials Science, Japan Advanced Institute of Science and Technology \\ (JAIST), Tatsunokuchi, Ishikawa 923-1292, Japan
}

(Received December 1, 1997)

\begin{abstract}
Heating measurements of crystallized poly( $\varepsilon$-caprolactone)-block-polybutadiene (PCL- $b$-PB) copolymers were performed using synchrotron small-angle X-ray scattering (SR-SAXS) to study the melting behavior of crystalline-amorphous diblock copolymers. The strong SAXS intensity peak, originating from the alternating structure of crystalline lamellae and amorphous layers (lamellar morphology), became broad in the course of melting and eventually a diffraction (or sharp intensity peak) arising from the microdomain structure emerged from this broad scattering peak, suggesting an interplay between the lamellar morphology and microdomain structure during melting. This is extremely different from the time-resolved SR-SAXS curve obtained during the crystallization of the microphase-separated PCL- $b$-PB, where the diffraction decayed steadily and the scattering peak (from the lamellar morphology) grew independently with increasing crystallization time. The melting behavior of PCL- $b$-PB evaluated from the SR-SAXS results was qualitatively compared with that of poly $(\varepsilon$-caprolactone) homopolymer (PCL). The long spacing of the lamellar morphology of PCL- $b$-PB, for example, decreased or remained constant during melting, whereas that of PCL increased slightly. The characteristics of the melting behavior of crystalline-amorphous diblock copolymers are discussed on the basis of the morphological reorganization from the lamellar morphology into the microdomain structure.
\end{abstract}

KEY WORDS Crystalline-Amorphous Diblock Copolymer / Melting Behavior / Time-Resolved SmallAngle X-Ray Scattering / Synchrotron Radiation /

Crystalline-amorphous diblock copolymers are an interesting system to study the complicated morphology formation in polymer systems because the crystallization of the constituent block leads to the morphological transformation from a microdomain structure into a lamellar morphology, an alternating structure of crystalline lamellae and amorphous layers. This lamellar morphology should be more complicated than that of crystalline homopolymers; the amorphous block has to be accommodated between the lamellae since both blocks are connected by the covalent bond so as to prevent the rejection of the amorphous block from the crystalline region. The equilibrium morphology formed in these crystalline-amorphous diblock copolymers was theoretically predicted, ${ }^{1,2}$ and the predictions agreed qualitatively with the recent experimental results performed with various crystalline-amorphous diblock copolymers. ${ }^{3-7}$ The crystallization behavior of various block copolymers has also been investigated by time-resolved small-angle $\mathrm{X}$-ray scattering using synchrotron radiation (SR-SAXS) and new aspects of crystallization were pointed out which could not be observed with crystalline homopolymers. $^{8-14}$

When crystallized block copolymers are heated at a constant rate, we can observe the melting process in which the lamellar morphology is reorganized into the microdomain structure, the reverse transformation during crystallization. The melting behavior of crystalline homopolymers is well known to be considerably different from the crystallization behavior, so that we can expect a new type of melting for these block copolymers which is not predicted from their crystallization. There are some experimental studies on the melting behavior of crystalline homopolymers observed by SR-SAXS. ${ }^{15-21}$
Chu et al., ${ }^{16}$ for example, studied the melting behavior of poly(aryl ether ether ketone) (PEEK) by SR-SAXS and found the steady increase of the long spacing during melting, which was attributed to the minor rearrangement of the lamellar morphology formed in PEEK under the nonequilibrium crystallization. There are, however, few studies on the melting behavior of crystalline-amorphous diblock copolymers investigated by SRSAXS.

In our earlier papers, ${ }^{7-10,22}$ we investigated quantitatively the crystallization behavior and final morphology of poly( $\varepsilon$-caprolactone)-block-polybutadiene (PCL- $b$ PB) copolymers by conventional SAXS, SR-SAXS, differential scanning calorimetry (DSC), and transmission electron microscopy (TEM), and elucidated the characteristics of the crystallization behavior and final morphology of PCL- $b$-PB. In the present study, we investigate the melting behavior of PCL- $b$-PB by SRSAXS. The SR-SAXS curves are qualitatively compared with those of PCL- $b$-PB during crystallization. In addition, the change in the long spacing, evaluated from the angular position of the SAXS intensity peak, was compared with that of poly( $\varepsilon$-caprolactone) homopolymer (PCL) during melting. From these experimental results, we try to find the characteristics of the melting behavior of crystalline-amorphous diblock copolymers and we discuss a cooperative morphological reorganization from the lamellar morphology into the microdomain structure during melting.

\section{EXPERIMENTAL}

\section{Materials \\ PCL- $b$-PB copolymers were synthesized by successive}

\footnotetext{
${ }^{\dagger}$ To whom correspondence should be addressed. (Phone: +81-761-51-1601, Fax: +81-761-51-1149, e-mail: nojima (ajaist.ac.jp).
} 
Table I. Characterization of polymers used in this study

\begin{tabular}{|c|c|c|c|c|c|c|c|c|c|}
\hline \multirow{2}{*}{ Notation } & \multirow{2}{*}{ Polymer } & \multirow{2}{*}{ Source } & \multirow{2}{*}{ Total $M_{w}{ }^{c}$} & \multirow{2}{*}{$M_{w} / M_{n}^{\mathrm{c}}$} & \multirow{2}{*}{$\frac{P C L: P^{d}}{\operatorname{vol}^{d} \%}$} & \multicolumn{3}{|c|}{ Microstructure of PB chain $/ \%$} & \multirow{2}{*}{$\frac{T_{\mathrm{m}}{ }^{\mathrm{e}}}{{ }^{\circ} \mathrm{C}}$} \\
\hline & & & & & & cis-1,4 & trans $-1,4$ & 1,2-Linkage & \\
\hline B7 & PCL- $b$-PB & $\mathrm{a}$ & 12,400 & 1.10 & $61: 39$ & 36 & 52 & 12 & 54 \\
\hline B11 & PCL- $b$-PB & $\mathrm{a}$ & 14,100 & 1.09 & $26: 74$ & 35 & 51 & 14 & 45 \\
\hline PCL1 & PCL & $\mathrm{b}$ & 11,000 & 1.57 & - & & - & & 55 \\
\hline
\end{tabular}

${ }^{a}$ Synthesized in our laboratory. ${ }^{\mathrm{b}}$ Obtained from Scientific Polymer Products, Inc. ${ }^{\mathrm{c}}$ Values relative to PS standards determined by GPC. ${ }^{\mathrm{d}}$ Determined by ${ }^{1} \mathrm{H}$ NMR. ${ }^{\mathrm{e}}$ Determined by DSC at a heating rate of $5^{\circ} \mathrm{C} \mathrm{min}^{-1}$.

anionic polymerizations in toluene under vacuum with $n$-butyllithium as the initiator. Details of the synthesis were described elsewhere. ${ }^{8,10}$ Two PCL- $b$-PB samples, designated $\mathrm{B} 7$ and $\mathrm{B} 11$ in our previous studies, ${ }^{7,10}$ were used in this study. The samples were characterized by gel permeation chromatography (GPC), and the PCL content in the copolymer was evaluated by ${ }^{1} \mathrm{H}$ NMR (Varian Gemini-200). The melting temperature $T_{\mathrm{m}}$ of the PCL block was measured by DSC (MAC Science Model 3100 or Perkin Elmer DSC7) at a heating rate of $5^{\circ} \mathrm{C}$ $\min ^{-1}$ and $2^{\circ} \mathrm{Cmin}^{-1}$, the latter is the same to the thermal history applied to the SR-SAXS experiments (Figure 1). The results of the molecular characterization are listed in Table I. B7 has a lamellar microdomain structure in the molten state and B11 has a hexagonallypacked cylindrical structure judging from the PCL content and the angular positions of small-angle X-ray intensity peaks. We also used a PCL, designated PCL1 in Table I, to compare the melting behavior with that of $\mathrm{B} 7$ and $\mathrm{B} 11$.

\section{Time-Resolved SAXS Measurements}

The crystallization and melting behaviors of B7, B11, and PCL1 were observed by time-resolved SAXS with synchrotron radiation (SR-SAXS). The experiment was performed at Institute of Materials Structure Science, Tsukuba, Japan (Photon Factory), with small-angle $\mathrm{X}$-ray equipment for solution (SAXES) installed on a beam-line BL-10C. The geometry was carefully checked by a chicken tendon collagen, which gives a set of sharp diffractions (up to 10th) corresponding to $65.3 \mathrm{~nm}$. Details of the optics and the instrumentation of the equipment were described elsewhere. ${ }^{23,24}$

The sample temperature was controlled by circulating water supplied by the programmable water bath. The thermal history applied to the sample was shown in Figure 1. First, the sample was annealed at temperatures above $T_{\mathrm{m}}$ for $c a .30 \mathrm{~min}$ to erase the previous thermal history, then quenched into the crystallization temperature $T_{\mathrm{c}}$ ranging from $20^{\circ} \mathrm{C}$ to $40^{\circ} \mathrm{C}$ by dropping the water temperature, kept at $T_{\mathrm{c}}$ for $30-100 \mathrm{~min}$ to observe the crystallization process by SR-SAXS, and finally heated at a constant heating rate $\left(\mathrm{ca} .2^{\circ} \mathrm{Cmin}^{-1}\right)$ until the sample was completely melted during which the melting process was observed by SR-SAXS. It took about $1 \mathrm{~min}$ to quench the molten sample to $T_{\mathrm{c}}$. The integrated SAXS intensity was also monitored during the crystallization and melting processes, which enabled us to proceed to the next step in the temperature program. The SAXS intensity was collected as an accumulation of the scattered intensity during $10 \mathrm{~s}$ both for the crystallization and melting processes.

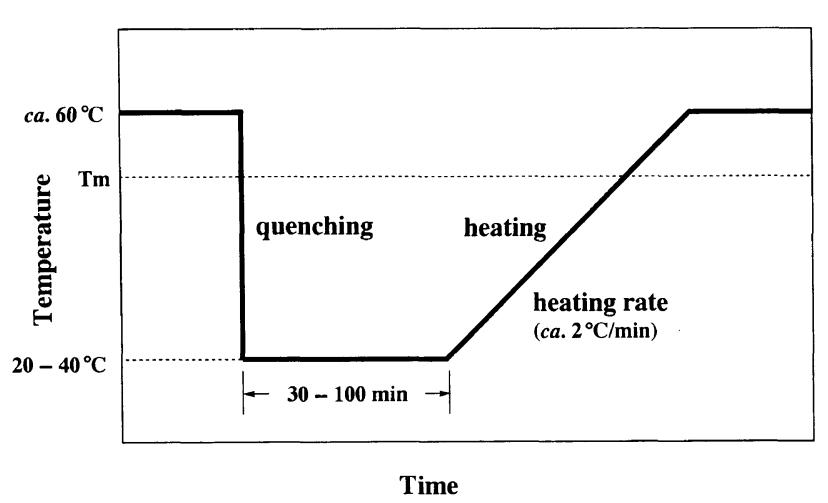

Figure 1. Thermal history applied to the sample during SR-SAXS measurements.

The SAXS intensity measured was corrected for the decrease of the ring current and background scattering. Since the optics of SAXES is of the point focusing, the scattered intensity was not corrected for the smearing effect by the finite cross section of the primary beam. ${ }^{24}$ The SAXS curve against wave number $s$ was finally obtained as a function of time $t$ during crystallization and as a function of temperature $T$ during melting, where $s$ is defined as,

$$
s=2 \sin \theta / \lambda
$$

where $2 \theta$ is the scattering angle and $\lambda$ is the $\mathrm{X}$-ray wave length used $(=0.1488 \mathrm{~nm})$.

\section{Analysis of SR-SAXS Data}

On the analysis of the time-resolved SAXS curves during melting, we focused our attention on the scattered intensity peaks arising from the lamellar morphology and the microdomain structure. That is, we obtained the temperature dependence of the peak position, peak intensity, and full width at half maximum (FWHM) of the peak. The intensity peak arising from the lamellar morphology was sufficiently apart from that arising from the microdomain structure in the crystallization process (see, Figures 5a and 6a). In the late stage of melting, however, these peaks approached each other to yield a combined bimodal scattering peak (see Figure 2). In order to separate these peaks on a computational method, each peak was approximated by the phenomenological functions; Gaussian $I_{\mathrm{G}}(s)$, Lorentzian $I_{\mathrm{L}}(s)$, and pseudoVoigt function $I_{\mathrm{V}}(s)$, a linear combination of $I_{\mathrm{G}}(s)$ and $I_{\mathrm{L}}(s)$ with a ratio of $\eta: 1-\eta$. They are defined by,

$$
I_{\mathrm{G}}(s)=\frac{2 A \sqrt{\ln 2}}{\sqrt{\pi} W} \exp \left[-\frac{4 \ln 2}{W^{2}}\left(s-s_{\max }\right)^{2}\right]
$$




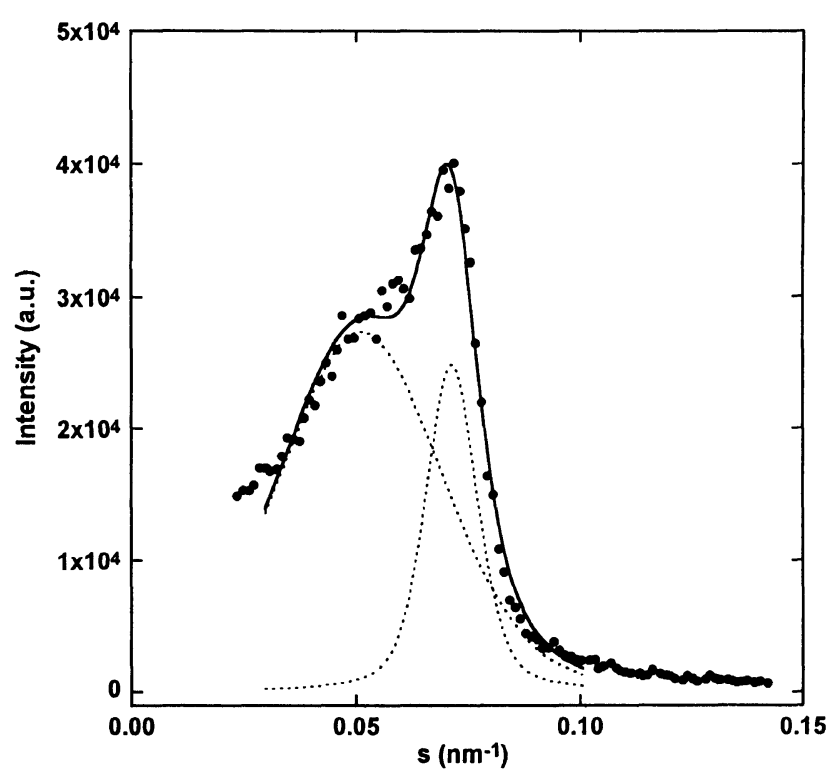

Figure 2. A typical fitting procedure of the experimental SAXS curve (closed circle) with two pseudo-Voigt functions (broken curves) for the scattering peaks arising from the microdomain structure (high $s$ peak) and lamellar morphology (low $s$ peak) to evaluate the characteristic parameters of each morphology. The solid curve represents the combined curve of two pseudo-Voigt functions.

$$
I_{\mathrm{L}}(s)=\frac{2 A}{\pi W}\left[1+\frac{4}{W^{2}}\left(s-S_{\max }\right)^{2}\right]^{-1}
$$

and

$$
I_{\mathrm{V}}(s)=\eta I_{\mathrm{G}}(s)+(1-\eta) I_{\mathrm{L}}(s)
$$

where, $W$ is the full width at half maximum of the peak, $A$ is proportional to the peak area, and $s_{\max }$ is the maximum value of $s$. These functions are usually used to approximate the diffraction intensities from crystalline materials in order to characterize each peak, ${ }^{25}$ and are empirically used with no theoretical basis.

Figure 2 shows a typical example of the fitting procedure by the two pseudo-Voigt functions for the scattering peaks from the lamellar morphology (lower $s$ peak) and the microdomain structure (higher $s$ peak) when these peaks come close to each other at the late stage of melting. The estimation of the best values of $A, W$, and $s_{\max }$ characterizing both peaks was relatively easy, and also the use of eq 2-4 did not make any significant difference in the resulting values, so that we used the pseudo-Voigt function (eq 4) both for the scattering peaks from the lamellar morphology and microdomain structure, though the use of eq 4 involved the evaluation of the additional parameter $(\eta)$.

\section{RESULTS}

\section{DSC Curves}

Figure 3 shows DSC thermograms measured with two heating rates $\left(2^{\circ} \mathrm{C} \mathrm{min}{ }^{-1}\right.$ and $\left.5^{\circ} \mathrm{Cmin}^{-1}\right)$ for PCL1 and $\mathrm{B} 7$ both crystallized at $40^{\circ} \mathrm{C}$. A single endothermic peak is observed for $\mathrm{B} 7$ and PCL1 at a heating rate of $5^{\circ} \mathrm{C}$ $\min ^{-1}$, whereas a small bump appears at a higher temperature in addition to the main peak with a heating rate of $2^{\circ} \mathrm{Cmin}^{-1}$. This bump always appeared for $\mathrm{B} 7$ and PCLl when the heating rate was lower than $2^{\circ} \mathrm{C}$

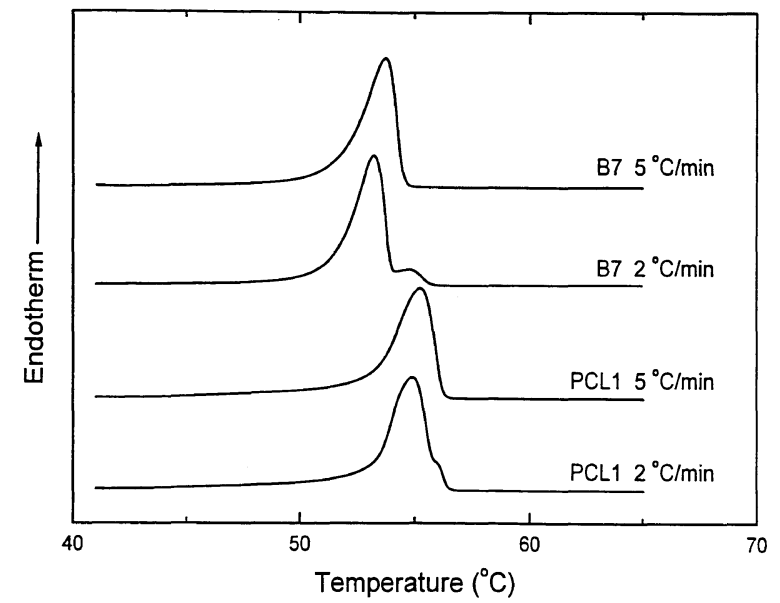

Figure 3. DSC thermograms for $\mathrm{B} 7$ and PCL1 crystallized at $40^{\circ} \mathrm{C}$. The heating rate $\left(2^{\circ} \mathrm{C} \mathrm{min}^{-1}\right.$ or $\left.5^{\circ} \mathrm{C} \mathrm{min}^{-1}\right)$ is indicated on each curve The DSC curves are not normalized by the amount of PCL chains existing in the system.

$\min ^{-1}$, and disappeared when the heating rate increased. The bimodal DSC melting curve is sometimes observed for crystalline homopolymers and usually attributed to the melting and recrystallization of thin lamellae kinetically formed under the nonequilibrium crystallization at $T_{\mathrm{c}}$. The bimodal curve of B7 shown in Figure 3 may originate from the same mechanism of PCLl since the heating rate dependence of the bump is similar to that of PCL1, but the quantitative investigation is necessary to confirm the reason for this bimodal endothermic curve of crystalline-amorphous diblock copolymers.

The melting temperature $T_{\mathrm{m}}$ of $\mathrm{B} 7$ is almost same to that of PCL1 and $T_{\mathrm{m}}$ of $\mathrm{B} 11$ is about $10^{\circ} \mathrm{C}$ lower than that of PCL1 (see Table I). A slight variation of $T_{\mathrm{m}}$ is usually observed in homopolymers crystallized from the melt, which is intimately related to the lamellar thickness determined at $T_{\mathrm{c}}$. The low $T_{\mathrm{m}}$ of $\mathrm{B} 11$, where the PCL block is extremely short compared with $\mathrm{B} 7$, may arise from the intrinsic effect of crystalline-amorphous diblock copolymers. That is, the PCL block has to be folded many times on the lamellar surface for the accommodation of the large PB block to result in a thin lamella. ${ }^{1,2}$ The unique morphology formed in crystalline-amorphous diblock copolymers will be discussed later on the basis of the theoretical prediction.

\section{Time-Resolved SAXS Curves}

A typical example of three-dimensional SAXS profiles obtained for B11 is depicted in Figure 4, where the thermal history shown in Figure 1 was applied. The crystallization process was measured as a function of time $t$ just after quenching the sample from the melt ( $c a$. $60^{\circ} \mathrm{C}$ ) into $T_{\mathrm{c}}$, and the melting process was measured as a function of temperature $T$ by heating the sample at a constant heating rate $\left(\mathrm{ca} .2^{\circ} \mathrm{C} \mathrm{min}^{-1}\right)$. The crystallization of $\mathrm{B} 7$ and $\mathrm{B} 11$ is a morphological reorganization process from a microdomain structure into a lamellar morphology and does not depend on a starting temperature, whereas the melting is a complicated process which may include several melting and recrystallization processes and depends intimately on the details of the lamellar morphology formed at $T_{\mathrm{c}}$ and also on the heating rate. 


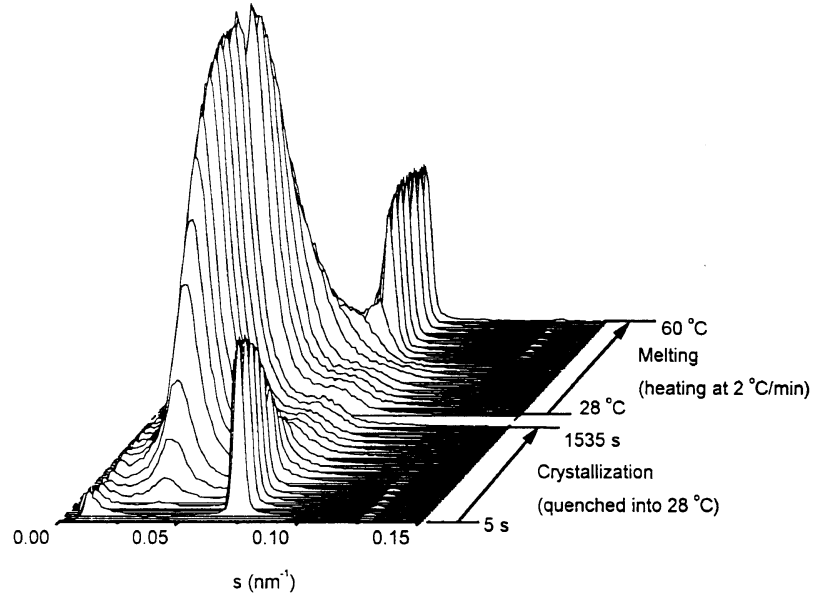

Figure 4. Overview of the time-resolved SR-SAXS curve obtained during the crystallization and melting of B11. The thermal history shown in Figure 1 was applied.

Melting in the present study is, therefore, not the reverse process of crystallization, so that we simply show the SR-SAXS curves during crystallization and melting (Figures 5 and 6) and we do not compare the details of both processes quantitatively.

Figure 4 shows clearly the reversible morphological transformation between the microdomain structure and lamellar morphology during thermal treatments, where the scattering peak arising from the microdomain structure (high $s$ peak) is sharp and weak compared with that from the lamellar morphology (low $s$ peak). The angular positions of these peaks suggest that the long spacing of the lamellar morphology is much larger than the repeating distance of the microdomain structure. ${ }^{7}$ This SAXS profile was reproducible when the thermal history shown in Figure 1 was repeatedly applied.

The SR-SAXS curve for PCL1 (not shown here) has no scattering peak at the initial stage of crystallization, since PCL1 is amorphous in the molten state. With increasing $t$, the lamellar morphology is formed and prevails in the system, therefore, the scattering peak appears and the intensity increases gradually with the peak position being constant. In the melting process of PCL1, on the other hand, the scattering peak is observed all through the heating until the PCL crystal melts, and the peak position shifts appreciably to the lower angle (i.e., the long spacing increases) with increasing $T$. These crystallization and melting processes of PCL1 observed by SR-SAXS agree qualitatively with the experimental results reported for other crystalline homopolymers. $^{15-21,26-29}$

In the case of $\mathrm{B} 11$ and $\mathrm{B} 7$, the crystallization and melting processes are extremely different from those of PCL1 as shown in Figures 5 and 6, where selected SAXS curves are presented successively to emphasize the morphological change during crystallization and melting. In the crystallization process of B11 (Figure 5a), for example, a diffraction intensity (or sharp peak intensity) arising from the microdomain structure decreases gradually with increasing $t$ and simultaneously a strong scattering from the lamellar morphology grows at a lower $s$. These two peaks seem to change independently and we can evaluate the $t$ dependence of two peaks suc-

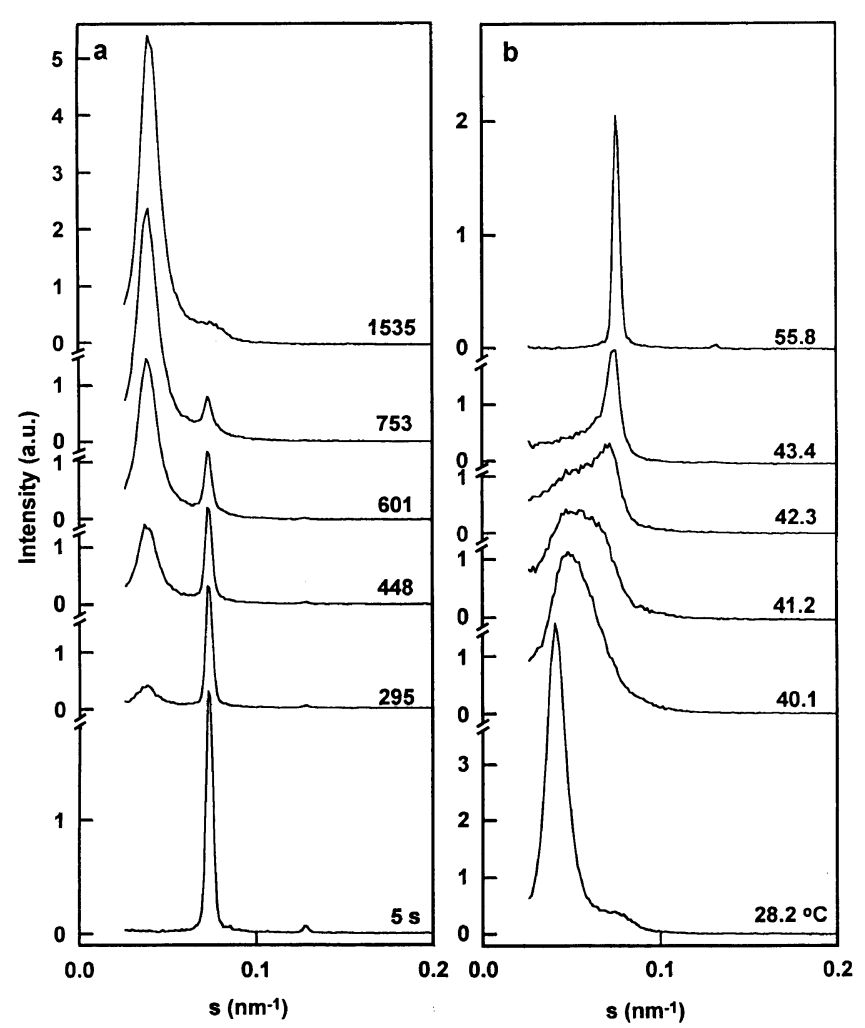

Figure 5. Selected time-resolved SAXS curves plotted against $s$ for B11 during crystallization (a) and melting (b). The SAXS curves for $5 \mathrm{~s}$ and $55.8^{\circ} \mathrm{C}$ are enlarged in magnitude by a factor of 2 for clarity.

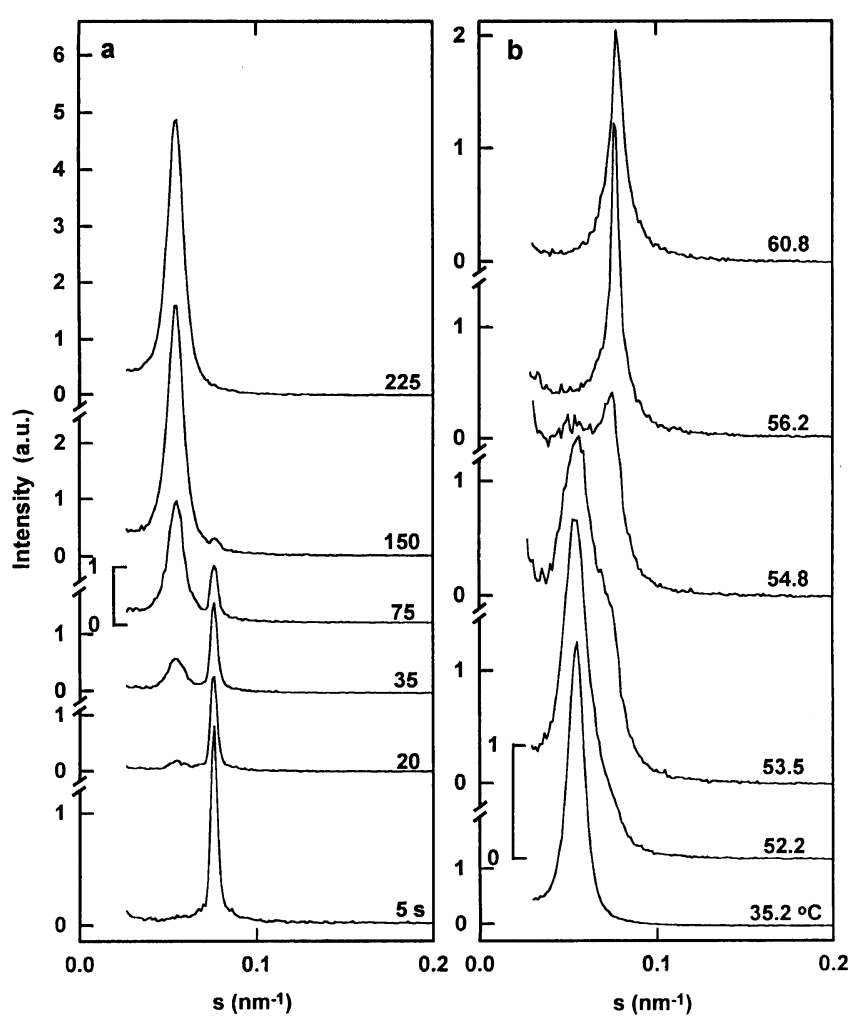

Figure 6. Selected time-resolved SAXS curves plotted against $s$ for B7 during crystallization (a) and melting (b). The SAXS curves for $5 \mathrm{~s}$, $53.5^{\circ} \mathrm{C}, 54.8^{\circ} \mathrm{C}, 56.2^{\circ} \mathrm{C}$, and $60.8^{\circ} \mathrm{C}$ are enlarged in magnitude by a factor of 2 for clarity.

cessfully, as shown in our previous studies, ${ }^{8,10}$ suggesting that the crystallization of the PCL block (and also the resulting lamellar morphology) is almost in- 


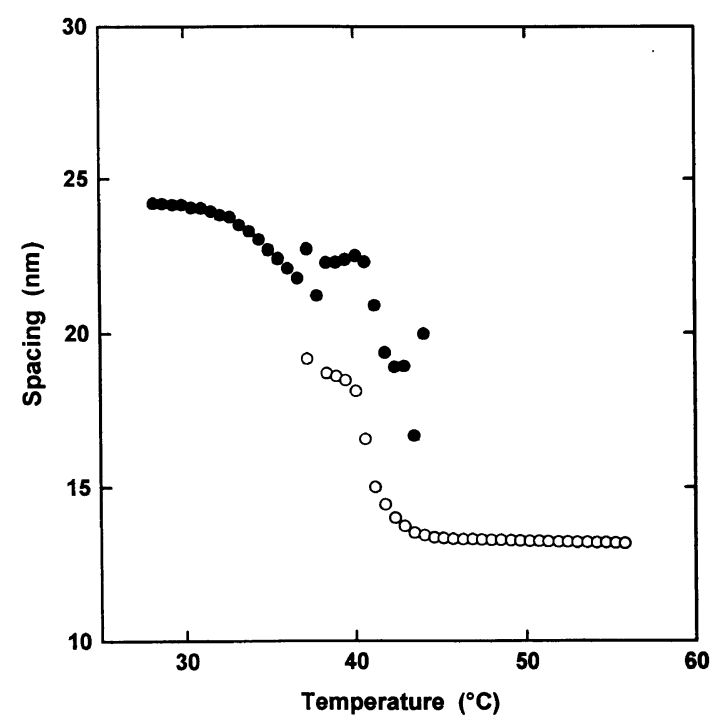

Figure 7. Spacings evaluated from the angular position of the SAXS intensity peaks arising from the microdomain structure $(O)$ and lamellar morphology (O) for B11 during melting.

dependent of the existing microdomain structure in the molten state. The melting process of B11 (Figure 5b) is, on the other hand, puzzling when it is compared with the crystallization process (Figure 5a). That is, the scattering peak arising from the lamellar morphology shifts to the higher $s$ and simultaneously the peak becomes broad during melting, and eventually the diffraction (arising from the microdomain structure) emerges from this broad scattering. Figures $5 \mathrm{~b}$ and $6 \mathrm{~b}$ suggest that the melting process proceeds by an interplay between two morphologies, that is, the microdomain structure is formed on the basis of the existing lamellar morphology at the late stage of melting. The possible melting mechanism of B7 and B11 will be discussed later.

\section{Melting Process of PCL- $b-P B$}

Since the crystallization behavior of PCL- $b$-PB has already reported quantitatively in our earlier publications, ${ }^{8-10}$ we only describe the details of the melting process of $\mathrm{B} 11$ in this section. The general picture of the melting processes of $B 7$ is the same to that of $B 11$, though there is a minor difference in the $T$ dependence of the long spacing of the lamellar morphology, as described later (Figure 10).

The long spacing of the lamellar morphology $L$ and the repeating distance of the microdomain structure $D$ during melting are shown in Figure 7 as a function of $T$. The value of $L$ decreases dramatically with increasing $T$ and seems to connect smoothly with $D$ at the intermediate stage of melting, though there is a considerable scattering of data points owing mainly to the fitting procedure (Figure 2). Figure 7 makes us speculate that the microdomain structure is formed just before melting with a minor morphological modification on the basis of the lamellar morphology which has changed appreciably during heating. This fact is reminiscent of the epitaxial growth recently observed in the morphological transformation in crystalline-amorphous diblock copolymers, ${ }^{30,31}$ where both the lamellar morphology and microdomain structure have an almost same repeating length and the transformation is achieved without a large

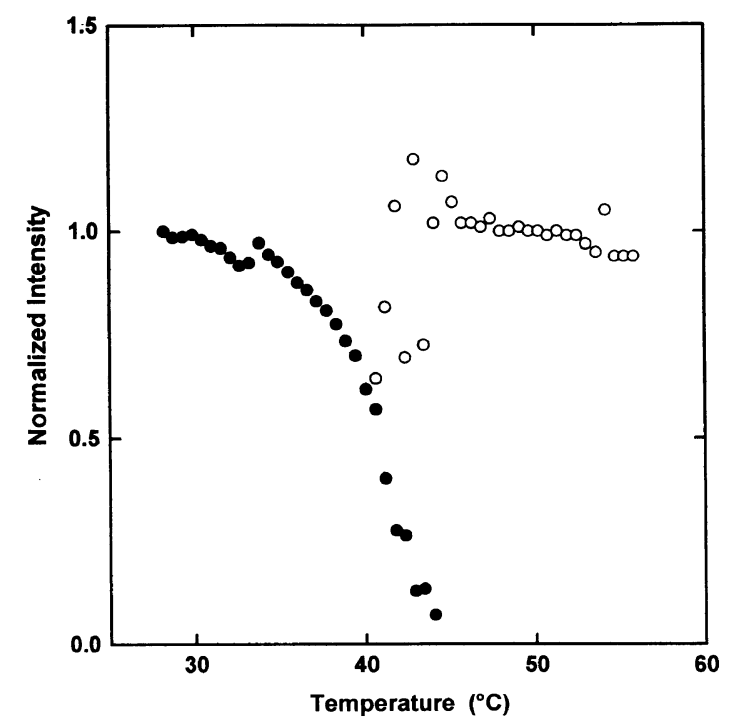

Figure 8. Normalized peak intensities arising from the microdomain structure $(\bigcirc)$ and lamellar morphology $(O)$ for B11 during melting. The peak intensities from the lamellar morphology are divided by the maximum value and those from the microdomain structure are normalized by an appropriate value because of the scattering of data points.

scale change in the morphology. The lamellar morphology once formed at $T_{\mathrm{c}}$ does not change during heating in their studies, which is significantly different from the present result for $\mathrm{B} 11$.

Figure 8 shows the $T$ dependence of the normalized peak intensities, i.e., peak intensities divided by their maximum values during melting. The peak intensity from the lamellar morphology decreases steeply with increasing $T$ and the diffraction appears when the lamellar morphology is almost destroyed. Figure 8 suggests that the lamellar morphology is transformed into the microdomain structure over a short temperature range, which is extremely different from the crystallization process, where the microdomain structure coexists with the lamellar morphology through a fairly long time. ${ }^{8}$

The FWHM, $W$, for these peaks is shown in Figure 9 as a function of $T$ during melting. The change of $W$ is dramatic, that is, each $W$ becomes extremely large at the intermediate stage of melting $\left(T \sim 40^{\circ} \mathrm{C}\right)$, which also suggests the cooperative morphological transformation from the lamellar morphology into the microdomain structure.

\section{Melting Processes of PCL-b-PB and PCL Homopolymer}

Figure 10 summarizes the $T$ dependence of the normalized long spacing of the lamellar morphology $L^{\prime}$, $L$ divided by the initial value, for B7, B11, and PCL1. The difference in $T_{\mathrm{c}}$ does not make any significant difference in this dependence, that is, the plots of $L^{\prime}$ against $T$ fall on a master curve for each sample irrespective of $T_{\mathrm{c}}$. The value of $L^{\prime}$ for PCL1 increases with increasing $T$ as usually observed in crystalline homopolymers, which is interpreted as the unfolding effect of multi-folded lamellae crystallized at $T_{\mathrm{c}}$. The value of $L^{\prime}$ for $\mathrm{B} 7$, on the other hand, remains constant and that for B11 decreases with increasing $T$, which are not reported so far for crystalline homopolymers and are considered to be characteristic of crystalline-amorphous diblock 


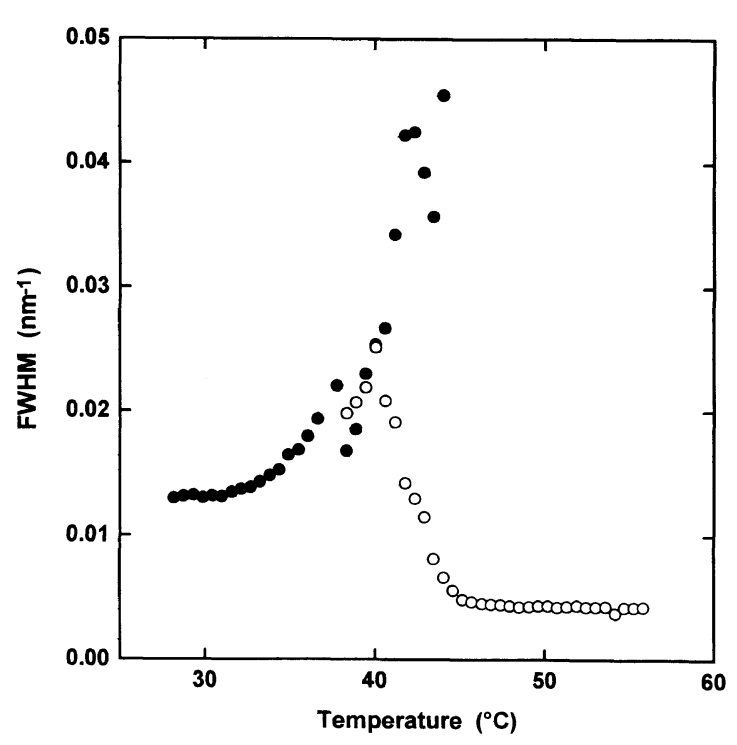

Figure 9. Full width at half maximum (FWHM) of the scattering peaks arising from the microdomain structure $(\mathrm{O})$ and lamellar morphology $(\bullet)$ for B11 during melting.

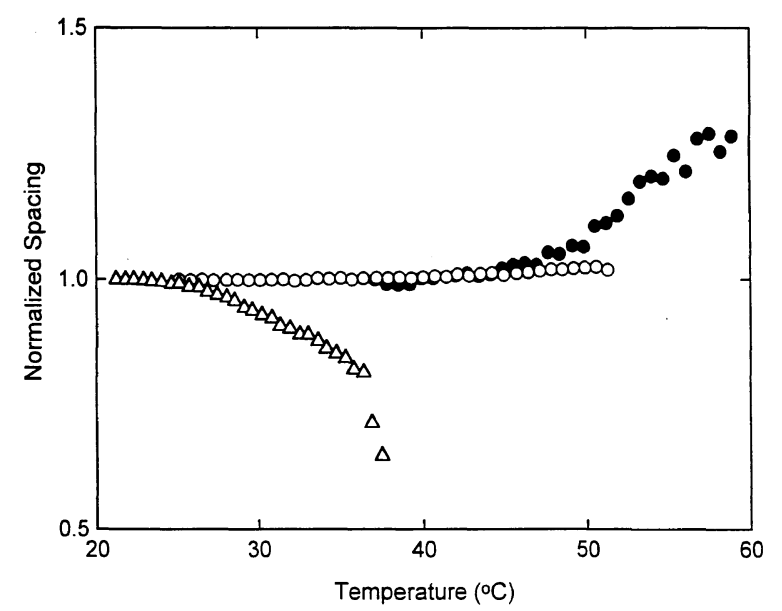

Figure 10. Temperature dependence of the normalized spacings of the lamellar morphology for B7 $(\bigcirc)$, B11 $(\triangle)$, and PCL1 $(\bullet)$. The spacings are divided by the initial values for each case.

copolymers. The essential difference in an equilibrium morphology between homopolymers and diblock copolymers will be responsible for the difference in the $T$ dependence of $L^{\prime}$ between PCL and PCL- $b$-PB shown in Figure 10, while the difference between B7 and B11 comes from the difference in the molecular structure of two copolymers, as discussed later.

\section{DISCUSSION}

Here, we consider the possible melting mechanism of crystalline-amorphous diblock copolymers from the SR-SAXS results obtained. The general picture of the melting process for crystalline homopolymers is well known since there are many experimental studies on the melting of homopolymers quantitatively investigated by various methods. ${ }^{32}$ Therefore, the comparison of the SR-SAXS results between PCL and PCL- $b$-PB may provide an important clue to understand the melting mechanism of diblock copolymers.

The SR-SAXS curve for the melting of PCL1 has an a

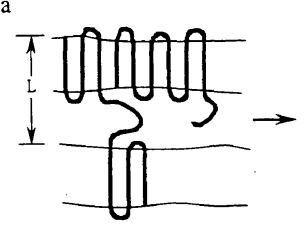

Lamellar Morphology

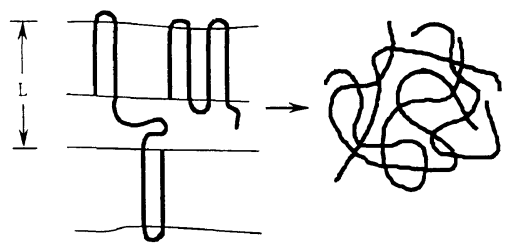

Amorphous State

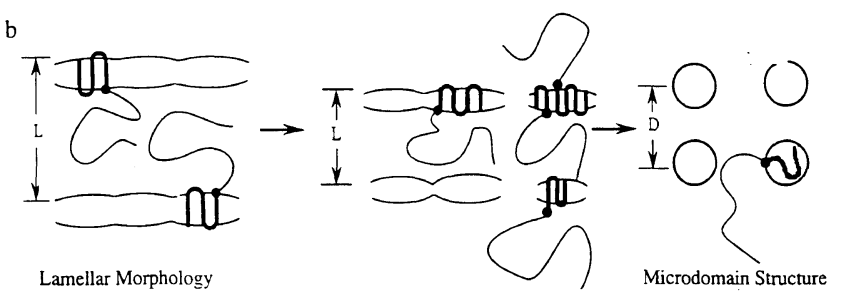

Figure 11. Schematic illustration showing the melting mechanism of PCL homopolymer (a) and PCL- $b$-PB (b).

intensity peak which shifts to the lower $s$ (i.e., larger $L$ ) with increasing $T$, as depicted in Figure 10. This increase of $L$ is usually attributed to the increase of both the lamellar thickness $l_{\mathrm{c}}$ and amorphous layer thickness $l_{\mathrm{a}}$ during melting. The increase of $l_{\mathrm{c}}$ and $l_{\mathrm{a}}$ is considered to arise mainly from the conformational modification of the crystallized homopolymer; the lamellar morphology formed under the nonequilibrium crystallization at $T_{\mathrm{c}}$ has a large number of folding on the lamellar surface to result in thinner lamellae with an appropriate folding free energy, and these lamellae thicken with increasing $T$ so as to reduce the folding number leading to the increase of $l_{\mathrm{a}}$ and $l_{\mathrm{c}}$. The schematic illustration of the melting mechanism of crystalline homopolymers is presented in Figure 11a. This picture is consistent with the present SR-SAXS result for PCL1 and also other SR-SAXS results reported so far. ${ }^{26-29}$

In the case of PCL- $b$-PB, $L$ decreases or remains constant with increasing $T$ depending on the molecular characteristics of the constituent diblock copolymers. The essential difference in the equilibrium morphology between crystalline homopolymers and crystalline-amorphous diblock copolymers will be responsible for the difference in the $T$ dependence of $L$ between PCL and PCL- $b$-PB (Figure 10). That is, the equilibrium morphology of crystalline homopolymers is an extended chain crystal with no folding and the lamellar morphology kinetically formed at $T_{\mathrm{c}}$ changes during heating so as to decrease the folding number toward the extended chain. The equilibrium morphology of crystallineamorphous diblock copolymers is, on the other hand, theoretically predicted to be determined by a cooperative effect of the stretching of the amorphous block (PB block) and the chain folding of the crystalline block (PCL block) on the lamellar surface. ${ }^{1,2}$ This is due to the additional restriction intrinsic to diblock copolymers arising from the covalent bond between crystalline and amorphous blocks; the space has to be offered to accommodate the amorphous block at the sacrifice of the increase in the folding number (and therefore, increase in the free energy) of the crystalline block. The increase of $l_{\mathrm{c}}$ (or decrease of the folding number) is, therefore, not necessarily favorable for the stability of the whole co- 
polymer, since the increase of $l_{\mathrm{c}}$ makes the amorphous block more stretched to yield the free energy increase of the amorphous block. This point is conclusively different from the melting of crystalline homopolymers.

In the melting process of B7 and B11 the microdomain structure and lamellar morphology interact with each other to yield the morphological transformation, that is, the microdomain structure is formed at the end of melting on the basis of the lamellar morphology, as found in Figures 7 and 8 . From these facts the melting mechanism of PCL- $b$-PB can be schematically illustrated in Figure $11 \mathrm{~b}$, where the melting of $\mathrm{B} 11$ is supposed; heating leads to the conformational change of B11 toward the equilibrium morphology with a larger folding number of the crystalline block to result in the decrease of lamellar thickness. The difference in the temperature dependence of $L$ between B7 and B11 can be qualitatively explained from the viewpoint of the cooperative effect working on the block copolymer. B1 1 has a large PB block compared with $\mathrm{B} 7$ and more space should be prepared to accommodate the PB block which expands with increasing $T$. The crystalline block, therefore, must have more folding numbers to be thinner rather than to be thicker; the free energy increase by stretching of the amorphous block overwhelms that by folding of the crystalline block. In the case of B7, both effects are balanced out and $L$ seems to remain constant irrespective of temperature.

The above speculation of the melting behavior of crystalline-amorphous diblock copolymers is based solely on the SR-SAXS results obtained in the present study. It is, therefore, necessary to elucidate the melting process quantitatively by other methods to understand the characteristics of the melting behavior of crystalline-amorphous diblock copolymers. The accumulation of the basic experimental results is necessary to understand the melting mechanism of crystalline-amorphous diblock copolymers.

Acknowledgments. This work was supported in part by Grants-in-Aid for Scientific Research (No. 08651072 and No. 09650992) from the Ministry of Education, Science, Sports and Culture of Japan, and has been performed under the approval of the Photon Factory Program Advisory Committee (Proposal No. 95G087).

\section{REFERENCES}

1. E. A. DiMarzio, C. M. Guttman, and J. D. Hoffman, Macromolecules, 13, 1194 (1980).

2. M. D. Whitmore and J. Noolandi, Macromolecules, 21, 1482 (1988).
3. K. C. Douzinas, R. E. Cohen, and A. F. Halasa, Macromolecules, 24, 4457 (1991).

4. R. Unger, D. Beyer, and E. Donth, Polymer, 32, 3305 (1991).

5. A. J. Lovinger, B. J. Han, F. J. Padden, and P. A. Mirau, J. Polym. Sci., Part B, 31, 115 (1993).

6. P. Rangarajan, R. A. Register, and L. J. Fetters, Macromolecules, 26, 4640 (1993).

7. S. Nojima, S. Yamamoto, and T. Ashida, Polym. J., 27, 673 (1995).

8. S. Nojima, K. Kato, S. Yamamoto, and T. Ashida, Macromolecules, 25, 2237 (1992).

9. S. Nojima, H. Nakano, and T. Ashida, Polym. Commun., 34, 4168 (1993).

10. S. Nojima, H. Nakano, Y. Takahashi, and T. Ashida, Polymer, 35, 3479 (1994).

11. P. Rangarajan, R. A. Register, D. H. Adamson, L. J. Fetters, W. Bras, S. Naylor, and A. J. Ryan, Macromolecules, 28, 1422 (1995).

12. A. J. Ryan, I. W. Hamley, W. Bras, and F. S. Bates, Macromolecules, 28, 3860 (1995).

13. P. Rangarajan, R. A. Register, L. J. Fetters, W. Bras, S. Naylor, and A. J. Ryan, Macromolecules, 28, 4932 (1995).

14. Y. W. Yang, S. Tanodekaew, S. M. Mai, C. Booth, A. J. Ryan, W. Bras, and K. Viras, Macromolecules, 28, 6029 (1995).

15. P. Schouterden, M. Vandermarliere, C. Riekel, M. H. J. Koch, G. Groeninckx, and H. Reynaers, Macromolecules, 22, 237 (1989).

16. B. S. Hsiao, K. H. Gardner, D. Q. Wu, and B. Chu, Polymer, 34, 3996 (1993).

17. K. N. Kruger and H. G. Zachmann, Macromolecules, 26, 5202 (1993).

18. W. J. O'Kane, R. J. Young, A. J. Ryan, W. Bras, G. E. Derbyshire, and G. R. Mant, Polymer, 35, 1352 (1994).

19. R. K. Verma, V. Velikov, R. G. Kander, H. Marand, B. Chu, and B. S. Hsiao, Polymer, 37, 5357 (1996)

20. F. J. M. Rodriguez, P. J. Phillips, and J. S. Lin, Macromolecules, 29, 7491 (1996).

21. A. J. Ryan, J. L. Stanford, W. Bras, and T. M. W. Nye, Polymer, 38, 759 (1997).

22. S. Nojima, K. Hashizume, A. Rohadi, and S. Sasaki, Polymer, 38, 2711 (1997).

23. T. Ueki, Y. Hiragi, M. Kataoka, Y. Inoko, Y. Amemiya, Y. Izumi, H. Tagawa, and Y. Muroga, Biophys. Chem., 23, 115 (1985).

24. S. Nojima, K. Kato, M. Ono, and T. Ashida, Macromolecules, 25, 1922 (1992).

25. G. Will, M. Bellotto, W. Parrish, and M. Hart, J. Appl. Crystallogr., 21, 183 (1988).

26. S. Nojima, H. Tsutsui, M. Urushihara, W. Kosaka, N. Kato, and T. Ashida, Polym. J., 18, 451 (1986).

27. R. Gehrke, C. Riekel, and H. G. Zachmann, Polymer, 30, 1582 (1989).

28. J. Wang, M. Alvarez, W. Zhang, Z. Wu, Y. Li, and B. Chu, Macromolecules, 25, 6943 (1992).

29. B. S. Hsiao, K. H. Gardner, D. Q. Wu, and B. Chu, Polymer, 34, 3986 (1993)

30. I. W. Hamley, J. P. A. Fairclough, N. J. Terrill. A. J. Ryan, P. M. Lipic, F. S. Bates, and E. T. Andrews, Macromolecules, 29, 8835 (1996).

31. A. J. Ryan, J. P. A. Fairclough, I. W. Hamley, S. M. Mai, and C. Booth, Macromolecules, 30, 1723 (1997).

32. B. Wunderlich, "Macromolecular Physics. 3. Crystal Melting," Academic Press, New York, N.Y., 1980. 